\title{
Freezing transition of the vortex liquid in a layered superconductor
}

\author{
Bahman Davoudi ${ }^{\mathrm{a}}$, Mohammad Kohandel ${ }^{\mathrm{a}, \mathrm{b}, *}$, Bilal Tanatar ${ }^{\mathrm{c}}$ \\ a Institute for Studies in Theoretical Physics and Mathematics, Tehran 19395-5531, Iran \\ ${ }^{\mathrm{b}}$ Department of Physics, Faculty of Science, Shahid Rajaee University, Tehran 16788, Iran \\ c Department of Physics, Bilkent University, Bilkent, Ankara 06533, Turkey
}

Received 1 August 2000; accepted 11 September 2000

\begin{abstract}
The self-consistent theory of Singwi, Tosi, Land, and Sjölander is used to describe the liquid-solid phase in a layered superconductor in the limit of weak interplane correlation effects as would be applicable to BSCCO materials. We calculate the local-field corrections, static structure factor, and pair correlation function and compare our results with other methods. The Hansen-Verlet criterion is used to estimate the freezing temperature of the vortex system. (C) 2001 Elsevier Science B.V. All rights reserved.
\end{abstract}

\section{Introduction}

There is currently much effort to understand the nature of vortex phases in high temperature superconductors [1,2]. In the classical (Abrikosov) picture of type-II superconductors, flux lines (FL) penetrate a clean material for fields $H>H_{\mathrm{cl}}$, to form a triangular lattice. This mean field phase diagram is modified by inclusion of the strong thermal fluctuations, leading to a thermodynamic phase transition in which the flux lattice melts to a liquid of FL [1-4]. It has now become possible to

\footnotetext{
${ }^{*}$ Corresponding author. Present address: Department of Physics, University of Waterloo, Waterloo, Ont., Canada, N2L 3G1. Tel.: +1-519-888-4567; fax: +1-519-746-8115.

E-mail address: kohandel@gandalf.uwaterloo.ca (M. Kohandel).
}

observe the melting transition experimentally. The indirect observations $[5,6]$ based on the resistivity measurements, and recent experiments [7-11] based on the observation of a jump in the magnetization or the latent heat show a first-order vortex lattice melting transition. On the other hand, vortex lattice melting has been studied theoretically using various approximations. Early works using the renormalization group [12] or density functional theory [13] have indicated a first-order transition. Elastic theory combined with the Lindemann criterion produce a melting line in good agreement with the experimental observations $[14,15]$. There is also interest in numerical simulations of FL systems [16-21] partly because of the relevance to quantum melting transition.

We study in this paper the freezing transition of the flux liquid (or equivalently, the melting of the flux lattice) in highly anisotropic high $T_{\mathrm{c}}$ 
superconductors, in the limit of weak interplane correlation effects. The pancake vortices in layered superconductors are described by interactions of electromagnetic nature and Josephson type. In the limit of infinite anisotropy, the superconducting layers are electronically decoupled or that the contribution to the FL energy arising out of the interlayer Josephson coupling is zero [22]. The physical picture is that of a system of pancake vortices confined to move on superconducting layers and interacting through an anisotropic pair potential. The only parameters that enter the theory are material parameters (such as, the London penetration depth, interlayer spacing for the layered superconductor, etc.). In this context, to describe the properties of the FL liquid we apply the self-consistent theory of Singwi, Tosi, Land and Sjölander (STLS) [23,24] to this model. Since the boson analogy introduced by Nelson [3,4] assumes a vortex interaction local in time, we believe that a classical approach using liquid state theories such as the hypernetted-chain approximation [13] or the STLS approximation would capture the anisotropic nature of the interaction better. The STLS approximation relies on the dielectric formulation of the many-body problem and consists of the decoupling of the two-body distribution function in terms of the pair correlation function, which leads to an expression for the local-field correction in terms of the static structure factor. The theory allows us to calculate the equilibrium correlation functions of the system in the liquid phase, such as the pair correlation function and the static structure factor for different magnetic field strengths and temperatures. The results are often compared with the density functional results $[22,25,26]$ and available Monte Carlo simulations [16-18], from which good agreement is obtained.

The behavior of the correlation functions may be used to study the phases of vortex lines. It is well known that the oscillatory behavior in the pair correlation function indicate the building up of the local correlations which drive the freezing transition to the solid phase or more generally to an ordered phase. Therefore, the phase transition can be detected by looking at the behavior of pair correlation function at a fixed temperature (magnetic field) and varying magnetic field (tempera- ture). The liquid-solid transition can also be observed using the static structure factor. Appearance of the larger peaks in the structure factor resembles the onset of a phase transition.

The rest of this paper is organized as follows: In Section 2, we present the model for the intervortex interaction and the basic equations of the STLS method. The numerical results are presented and discussed in Section 3, and the conclusions appear in Section 4.

\section{Theory}

In a layered system in the limit of zero interlayer Josephson coupling, the pancake vortices can be treated as point like classical particles restricted to move in the planes. The Fourier transform of the intervortex interaction is given by the threedimensional (3D) anisotropic potential [27],

$\beta V(\vec{k})=\frac{\Gamma \lambda^{2}\left[k^{2}+\left(4 / d^{2}\right) \sin ^{2}\left(k_{z} d / 2\right)\right]}{k^{2}\left[1+\lambda^{2} k^{2}+4\left(\lambda^{2} / d^{2}\right) \sin ^{2}\left(k_{z} d / 2\right)\right]}$,

where $\vec{k}=\left(k, k_{z}\right)$ is the wave vector, $\lambda$ is the planar London penetration depth and $\Gamma=\beta d \phi_{0}^{2} / 4 \pi \lambda^{2}$ is a dimensionless strength parameter where $\phi_{0}$ is the flux quantum, $d$ is the distance between the planes and $\beta=1 / k_{\mathrm{B}} T$. The two dimensional (2D) pair potentials are repulsive for pancakes residing in the same layer and attractive for pancakes in different layers. However, the interlayer interaction is always weaker than intralayer interaction (e.g. for BSCCO it is weaker by a factor of 100 [22]).

Given this potential of interaction between vortices, the evaluation of equilibrium properties of the vortex liquid can be reduced to a problem in 3D classical system. Hence, one may find the basic quantities, such as the static structure factor, the pair correlation function and the spectrum of the excitation energies using the classical STLS approximation. We thus briefly discuss the basic equations of this approach. A similar method was recently used for describing the correlations in the layered electron gas [28].

The STLS self-consistent method consists of the determination of the density-density response 
function $\chi(q, \omega)$ which is given in terms of a localfield correction $G(q)$ dependent on the static structure factor

$\chi\left(q, q_{z}, \omega\right)=\frac{\chi_{0}^{2 \mathrm{D}}(q, \omega)}{1-\psi\left(q, q_{z}\right) \chi_{0}^{2 \mathrm{D}}(q, \omega)}$,

where $\chi_{0}^{2 \mathrm{D}}(q, \omega)$ is the $2 \mathrm{D}$ non-interacting densitydensity response function and the effective field is given as $\psi\left(q, q_{z}\right)=V\left(q, q_{z}\right)\left[1-G\left(q, q_{z}\right)\right]$. The local-field correction $G\left(q, q_{z}\right)$ describing the correlation effects is defined as [28]

$$
\begin{aligned}
G\left(q, q_{z}\right)= & \frac{d}{n(2 \pi)^{3}} \int_{-\pi / d}^{\pi / d} \mathrm{~d} k_{z} \int \mathrm{d}^{2} k \frac{V\left(k, k_{z}\right)}{V\left(q, q_{z}\right)} \\
& \times \frac{\vec{k} \cdot \vec{q}+k_{z} q_{z}}{q^{2}+q_{z}^{2}}\left[1-S\left(k-q, k_{z}-q_{z}\right)\right],
\end{aligned}
$$

where $n$ is the 2D density of vortices defined by $n=B / \phi_{0}$ and the static structure factor is given by

$S\left(q, q_{z}\right)=\frac{1}{1+\beta n \psi\left(q, q_{z}\right)}$.

Thus, we need to solve the equations for $G\left(q, q_{z}\right)$ and $S\left(q, q_{z}\right)$ self-consistently for fixed parameters. However, the above set of equations consist of a $3 \mathrm{D}$ integral and are somewhat difficult for numerical evaluation in an iterative scheme. Since the interlayer interactions are weaker than intralayer interactions, to simplify the calculations, we assume that the correlation effects between the planes can be neglected, and write the effective interaction as

$\psi\left(q, q_{z}\right)=V\left(q, q_{z}\right)-V^{2 \mathrm{D}}(q) G^{2 \mathrm{D}}(q)$,

where

$V^{2 \mathrm{D}}(q)=\frac{\Gamma \lambda^{2}}{1+\lambda^{2} k^{2}}$

is obtained by the large $d$ limit of anisotropic interaction $V\left(q, q_{z}\right)$. A similar approximation for layered electron structures was introduced by Miesenböck and Tosi [29]. Neglect of interlayer correlations in electronic systems may be questionable, but for layered BSCCO systems is fully justified. In this case, we need to calculate a simpler local-field correction
$G^{2 \mathrm{D}}(q)=\frac{-1}{n} \int \frac{\mathrm{d}^{2} k}{(2 \pi)^{2}} \frac{\vec{q} \cdot \vec{k}}{q^{2}} \frac{V^{2 \mathrm{D}}(k)}{V^{2 \mathrm{D}}(q)}[S(q-k)-1]$,

where $S(q)$ is the planar static structure factor and is related to the full $S\left(q, q_{z}\right)$ by

$S\left(q, q_{z}\right)=\sum_{m} S(q) \mathrm{e}^{-\mathrm{i} m q_{z} d}$.

Inverting the above relation, we obtain

$S(q)=S_{0}(q)=\int_{-\pi / d}^{\pi / d} \frac{\mathrm{d} q_{z}}{(2 \pi / \mathrm{d})} S\left(q, q_{z}\right)$,

where the $S\left(q, q_{z}\right)$ is defined by Eqs. (2.4) and (2.5).

In Section 3, we present the results of the numerical calculations for various physical quantities.

\section{Numerical results and discussion}

We numerically solve the set of self-consistent STLS equations with the potential defined in Eq. (2.1), and find the static structure factor and the pair correlation function. All the calculations are performed by choosing the typical parameters of BSCCO. The London penetration depth is taken to have Ginzburg-Landau temperature dependence $\lambda(T)=\lambda_{0} /\left(1-\left(T / T_{\mathrm{c}}\right)\right)^{1 / 2}$ with $\lambda_{0}=1000 \AA$, $T_{\mathrm{c}}=90 \mathrm{~K}$ and $d=15 \AA$.

The wave vector dependence of the $3 \mathrm{D}$ static structure factor is rather smooth and does not have any peaks when the system is in the liquid state, say at high temperature. However, if one plots the 3D static structure factor for $B=10 \mathrm{~T}$ and at low temperature such as $T=30 \mathrm{~K}$, as in Fig. 1, a peak structure in $S\left(q, q_{z}\right)$ appears. This indicates that as the temperature decreases the system develops stronger correlations and it is going to undergo a phase transition from the liquid phase into the solid-like ordered phase. The $z$-dependence of the static structure factor for low temperature indicates strong correlations in this direction in contrast to the high temperature results.

The scenario of approaching the "freezing" transition can also be discussed by plotting the 2D static structure factor. The results are displayed in Fig. 2 for a fixed magnetic field and different 


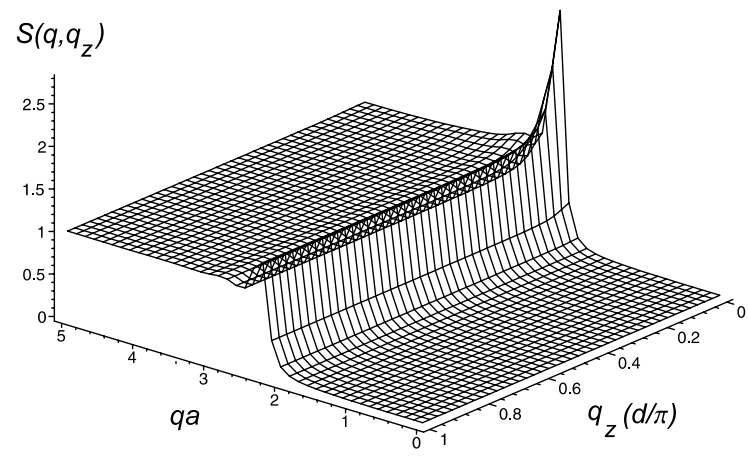

Fig. 1. The 3D static structure factor $S\left(q, q_{z}\right)$ for $B=10 \mathrm{kG}$ and $T=30 \mathrm{~K}$. The wave vector $q$ is scaled by $a$, the average distance between vortices, i.e. $n=1 /\left(\pi a^{2}\right)$.

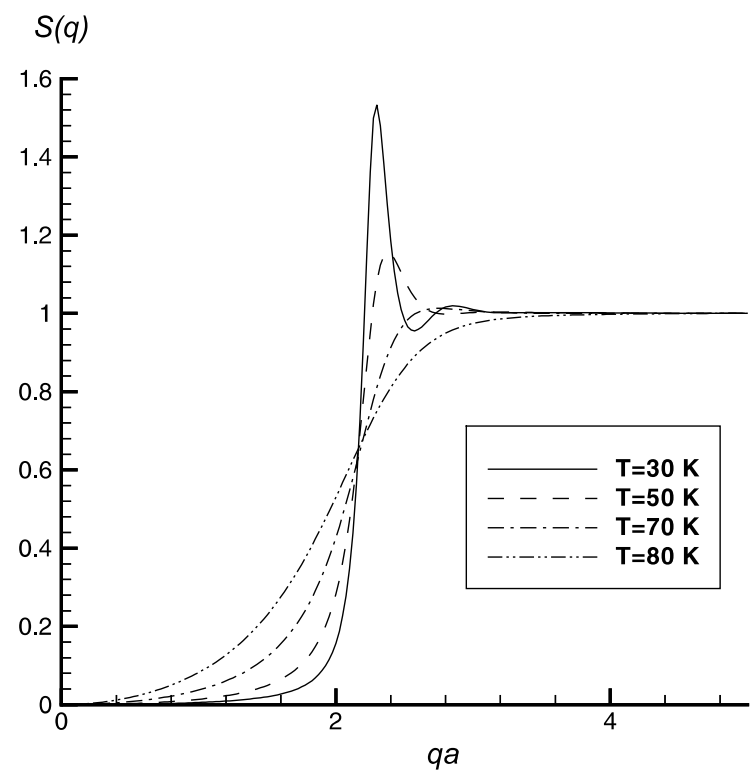

Fig. 2. The $2 \mathrm{D}$ static structure factor for $B=10 \mathrm{kG}$ and different temperatures.

temperatures. Fig. 2 demonstrates the very expected behavior of the static structure factor, that is the first peak of the $S(q)$ increasing with decreasing temperature, and shows a tendency toward the formation of an ordered phase. One can also observe the "freezing" transition by fixing the temperature and varying the magnetic field. Choosing $T=30 \mathrm{~K}$, we have calculated $S(q)$ for different magnetic fields, as shown in Fig. 3, which again indicates a transition into a more structured phase.

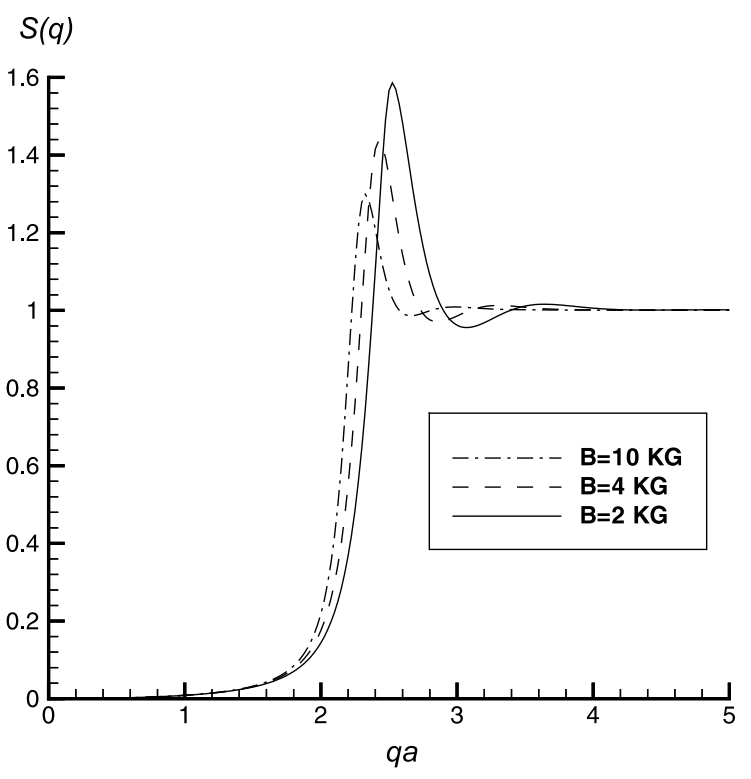

Fig. 3. The $2 \mathrm{D}$ static structure factor for $T=30 \mathrm{~K}$ and different magnetic fields.

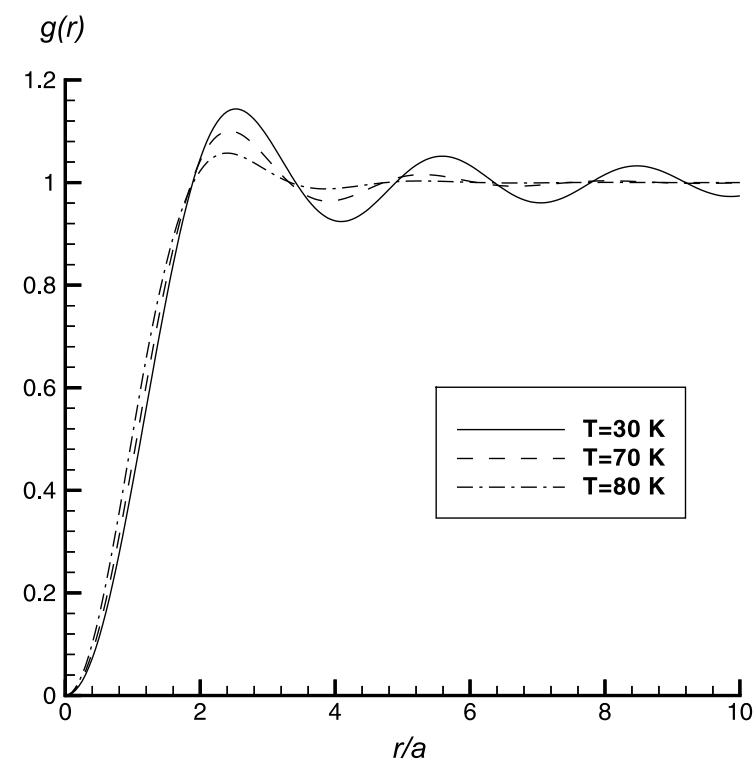

Fig. 4. The pair correlation function for $B=10 \mathrm{kG}$ and different temperatures.

The phase transition can be further studied by looking at the behavior of the pair correlation function. The results of $g(r)$ for $B=10 \mathrm{kG}$ and different temperatures are plotted in Fig. 4. It is 
generally regarded that the increased oscillations in $g(r)$ can be taken as a signature of the onset of an ordered phase developing. Therefore, Fig. 4 indicates a transition from the liquid phase to an ordered, say the solid phase with decreasing temperature. The results of the pair correlation function and static structure factor are in good qualitative agreement with the expected results of the phase diagram of the vortex system.

To obtain an estimation of the $B-T$ phase diagram, we use the Hansen-Verlet freezing criterion [30] which have been used by many authors [25,31], which states that the liquid freezes when the peak of the structure factor reaches a critical value. More specifically, the liquid freezes when the first peak of the structure factor reaches the value 2.85 in a $3 \mathrm{D}$ classical system and of the order of 5.5 for a 2D plasma [30]. We have calculated $S_{\max }\left(q, q_{z}=0\right)$, and found that the transition temperatures are compatible with the expected results of the phase diagram [1] and recent Monte Carlo simulations [16-18]. The maximum of $S\left(q, q_{z}=0\right)$ increases (for a fixed $B$ ) with decreasing $T$. By comparing with the results of the Monte Carlo simulations, we assume that the freezing transition appears when the peak of the structure factor reaches the value $S_{\max }\left(q, q_{z}=0\right) \approx 5$, and extracted the freezing temperature based on this criterion. The resulting $B-T$ phase diagram is plotted in Fig. 5 for different $\lambda$. The results show that by increasing the penetration depth the phase boundary shifts down as expected from the elasticity theory. [1] The result of phase diagram is in good qualitative agreement with the known estimations of the vortex matter phase diagram as obtained from Monte Carlo simulations [17,18] and other liquid state theory calculations $[13,22$, $25,26]$.

\section{Conclusions}

In this paper we have studied the phase diagram of a layered superconductor BSCCO within the classical STLS approximation. We have self-consistently determined the static structure factor and pair correlation function for various values of the

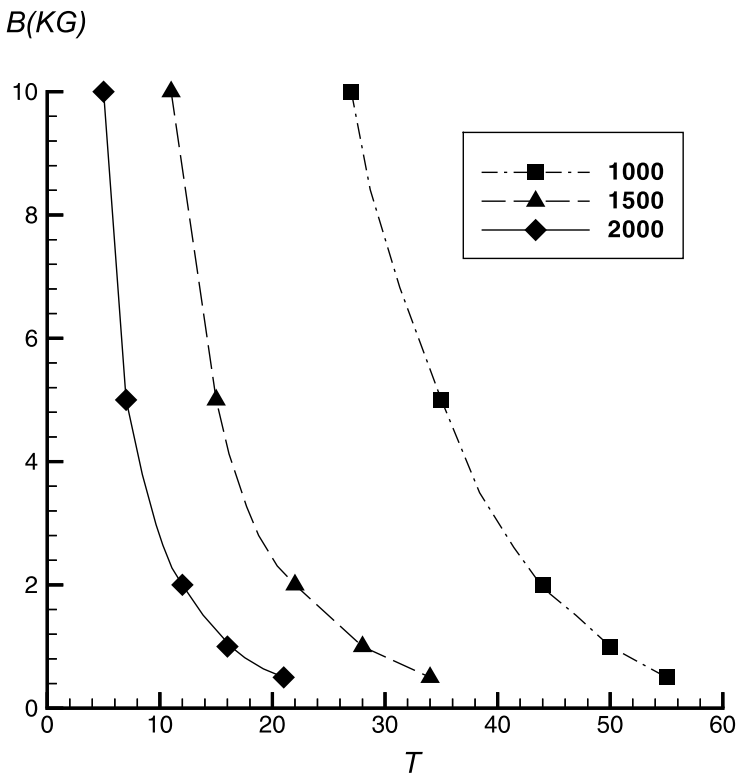

Fig. 5. The $B-T$ phase diagram of the FL system for different $\lambda$.

temperature and the magnetic field. We have discussed how the static correlation functions may be utilized to describe the nature of the vortex matter phases. The results were in good qualitative agreement with the expected phase diagram of the FL liquids. For the estimation of the $B-T$ phase diagram quantitatively, we invoked the behavior of the $S_{\max }\left(q, q_{z}=0\right)$ combined with Hansen-Verlet criterion. Our results are in good qualitative agreement with Monte Carlo simulations and classical hypernetted-chain approximation calculations. It would be interesting to extend our calculations to the case where the influence of disorder is taken into account.

\section{Acknowledgements}

B.D. and M.K. acknowledge support from the Institute for Studies in Theoretical Physics and Mathematics, Tehran, Iran. The work of B.T. is partially supported by the Scientific and Technical Research Council of Turkey (TUBITAK) under grant no. TBAG-1662 and NATO under grant no. SfP971970. 


\section{References}

[1] G. Blatter, M.V. Feigel'man, V.B. Geshkenbein, A.I. Larkin, V.M. Vinokur, Rev. Mod. Phys. 66 (1994) 1125.

[2] E.H. Brandt, Rep. Prog. Phys. 58 (1995) 1465.

[3] D.R. Nelson, Phys. Rev. Lett. 60 (1988) 1973.

[4] D.R. Nelson, H.S. Seung, Phys. Rev. B 39 (1989) 9153.

[5] H. Safar, P.L. Gammel, D.A. Huse, D.J. Bishop, J.P. Rice, D.M. Ginsberg, Phys. Rev. Lett. 69 (1992) 824.

[6] M. Charalambous, J. Chaussy, P. Lejay, Phys. Rev. B 45 (1992) 5091.

[7] E. Zeldov, D. Majer, M. Konczykowski, V.B. Geshkenbein, V.M. Vinokur, H. Shtrikman, Nature 375 (1995) 373.

[8] U. Welp, J.A. Fendrich, W.K. Kwok, G.W. Crabtree, B.W. Veal, Phys. Rev. Lett. 76 (1996) 4809.

[9] A. Schilling, et al., Nature 382 (1996) 791.

[10] M. Roulin, A. Junod, E. Walker, Science 273 (1996) 1210.

[11] A. Oral, J.C. Barnard, S.J. Bending, I.I. Kaya, S. Ooi, H. Taoka, T. Tamegai, M. Henini, Phys. Rev. Lett. 80 (1998) 3610 .

[12] E. Brezin, D.R. Nelson, A. Thiaville, Phys. Rev. B 31 (1985) 7124.

[13] S. Sengupta, C. Dasgupta, H.R. Krishnamurty, G.I. Menon, T.V. Ramakrishnan, Phys. Rev. Lett. 67 (1991) 3444.

[14] A. Houghton, R.A. Pelcovits, A. Subdo, Phys. Rev. B 40 (1989) 6763
[15] G. Blatter, V.B. Geshkenbein, A.I. Larkin, H. Nordborg, Phys. Rev. B 54 (1996) 72.

[16] W.R. Magro, D.M. Ceperley, Phys. Rev. Lett. 73 (1994) 826.

[17] H. Nordborg, G. Blatter, Phys. Rev. Lett. 79 (1997) 1925.

[18] H. Nordborg, G. Blatter, Phys. Rev. B 58 (1998) 14556.

[19] C. Bulutay, B. Tanatar, M. Tomak, Phys. Rev. B 57 (1998) 15197.

[20] E. Strepparola, R. Nifosi, M.P. Tosi, J. Phys.: Condens. Matter 10 (1998) 11645.

[21] B. Davoudi, M. Kohandel, Phys. Rev. B 61 (2000) 14727.

[22] G.I. Menon, C. Dasgupta, H.R. Krishnamurty, T.V. Ramakrishnan, S. Sengupta, Phys. Rev. B 54 (1996) 16192.

[23] K.S. Singwi, M.P. Tosi, R.H. Land, A. Sjölander, Phys. Rev. 176 (1968) 589.

[24] K.S. Singwi, M.P. Tosi, Solid State Phys. 36 (1981) 177.

[25] P.S. Cornoglia, C.A. Balsiero, Phys. Rev. B 61 (2000) 784.

[26] A. Kramer, J.E. Diaz-Herrera, Phys. Rev. B 58 (1998) 8755.

[27] M.V. Feigel'man, V.B. Geshkenbein, A.I. Larkin, Physica C 167 (1990) 177.

[28] W.B. Da Costa, U. De Freitas, N. Studart, Phys. Stat. Sol. (b) 206 (1998) 661

[29] H.M. Miesenböck, M.P. Tosi, Z. Phys. B 78 (1990) 255.

[30] J.P. Hansen, L. Verlet, Phys. Rev. 184 (1969) 151.

[31] A. Caillol, et al., J. Stat. Phys. 28 (1982) 325. 